\title{
Embodied cognition and theory of mind
}

\author{
Shannon Spaulding
}

\section{Introduction}

Social cognition is the ability to understand and interact with other agents. Traditionally, philosophers and psychologists have assumed that in order to understand and successfully interact with other people we must have a theory of mind, i.e., a theory about how mental states, such as beliefs, desires, and intentions, inform behavior and how behavior affects mental states. There are two main competing accounts of theory of mind: Theory Theory (TT) and Simulation Theory (ST).

TT holds that we explain and predict behavior by employing folk psychological theories about how mental states inform behavior. With our folk psychological theories, we infer from a target's behavior what his or her mental states probably are. From these inferences, plus the psychological principles in the theory connecting mental states to behavior, we predict the target's behavior.

ST, in contrast, holds that we explain and predict a target's behavior by using our own minds as a model. We imagine ourselves in the target's situation and figure out what our mental states would be and how we would behave if we were in the target's situation. We retrodictively simulate what the target's mental states could have been to cause the observed behavior, then we take the target's mental states in the form of pretend beliefs and pretend desires as input, run them through our own decision-making mechanism, take the resulting conclusion and attribute it to the target.

TT and ST disagree about how theory of mind works. TT contends that it is an information-rich theoretical process, whereas ST maintains that it is an information-poor simulational process. Though TT and ST proponents disagree about how theory of mind operates, they agree that social cognition requires theory of mind. There is an extensive empirical literature on theory of mind that aims at testing whether our theory of mind is theoretical or simulational in nature, when children develop theory of mind, and whether our theory of mind concepts are innate or learned.

According to embodied cognition, the philosophical and empirical literature on theory of mind is misguided. Embodied cognition rejects the idea that social cognition requires theory of mind. It regards the intramural debate between TT and ST as irrelevant, and it dismisses the empirical studies on theory of mind as ill conceived and misleading. Embodied cognition provides a novel deflationary account of social cognition that does not depend on theory of mind. In the next two sections, I shall describe embodied cognition's alternative to theory of mind and discuss three challenges it faces.

\section{Embodied Social Cognition}

Embodied cognition proponents reject the idea that social cognition is based on ascribing mental states to others. On their account, the capacity for more basic, non-mentalistic, interactive embodied practices underlies our ability to understand and interact with 
others. These interactive embodied practices consist in primary intersubjectivity and secondary intersubjectivity.

Primary intersubjectivity is the pre-theoretical, non-conceptual, embodied understanding of others that underlies and supports the higher-level cognitive skills posited in the theory of mind literature. It is "the innate or early developing capacity to interact with others manifested at the level of perceptual experience - we see or more generally perceive in the other person's bodily movements, facial gestures, eye direction, and so on, what they intend and what they feel" (Gallagher, 2005, p. 204). Primary intersubjectivity is manifested as the capacity for facial imitation and proprioceptive sense of one's body, the capacity to detect and track eye movement, to detect intentional behavior, and to read emotions from actions and expressive movements of others. Primary intersubjectivity consists in informational sensitivity and appropriate responsiveness to specific features of one's environment. It does not, embodied cognition theorists argue, involve representing those features. It simply requires certain practical abilities that have been shaped by selective pressures, e.g., being sensitive to certain bodily cues and facial expressions.

The development of secondary intersubjectivity occurs around age 1 , and it is marked by a move from one-on-one, immediate intersubjectivity to contexts of shared attention. In addition to tracking eye movement, detecting intentional behavior, and reading emotions, with the development of secondary intersubjectivity the child develops the capacity to communicate with others about objects and events in the environment. The child's interactions with caretakers begin to have reference to the things in their environment. At this stage, the child learns to follow gazes, point, and communicate with others about objects of shared attention. With secondary intersubjectivity, the child's capacity for social understanding is further developed, but according to embodied cognition this understanding is still non-mentalistic (Gallagher, 2005, p. 207).

Embodied cognition holds that these embodied intersubjective practices constitute our primary mode of social cognition(Gallagher, 2005; Hutto, 2008). Daniel Hutto claims, "Our primary worldly engagements are nonrepresentational and do not take the form of intellectual activity" (Hutto, 2008, p. 51). Theory of mind, it is argued, is a late developing, rarely used, specialized skill. The embodied practices constituted by primary and secondary intersubjectivity are developmentally fundamental. That is, in order to develop the capacity to have beliefs about others' mental states, one must first have a grasp of these basic embodied practices. Moreover, embodied intersubjectivity continues to be our principal mode of social interaction even in adulthood. Even as adults, our ordinary social interactions consist primarily in being sensitive to others' embodied practices. ${ }^{1}$

Of course, as adults we do have a capacity for more sophisticated social cognition. This is undeniable. However, according to embodied cognition, this capacity for more sophisticated social cognition does not involve theory of mind. As adults, our everyday social cognition consists only in these embodied practices and our knowledge of the social norms and behavioral scripts distinctive of our social environments. Daniel Hutto's Narrative Practice Hypothesis (NPH) is an embodied cognition-inspired account of how children develop knowledge of social norms and behavioral practices. It is not the only

\footnotetext{
${ }^{1}$ For a critical assessment of these ideas, see Spaulding (2010)
} 
possible embodied cognition account of sophisticated social cognition, but it is a particularly well-developed account.

NPH holds that the source of our capacity for sophisticated social cognition is direct encounters with folk psychological narratives, stories that exemplify the forms and norms of social interactions. Stories like Little Red Riding Hood and Goldilocks and the Three Bears are paradigmatic folk psychological narratives. The narratives provide exemplars of how agents act according to reasons in order to attain some goal. The child and her caretaker jointly attend to the narrative, and through guided interaction with a caretaker, the child becomes acquainted with forms and norms of acting for reasons. On this view, developing sophisticated social cognition consists in learning how to understand and provide reasons for actions. NPH is meant to be completely independent from theory of mind. Understanding the ways in which agents act for reasons does not consist in, nor does it depend on, attributing beliefs and desires to an agent in order to explain and predict the agent's behavior. Thus, our sophisticated capacity for social cognition does not consist in or depend on theory of mind (Gallagher \& Hutto, 2008).

$\mathrm{NPH}$ holds that to be proficient in giving reasons for actions, children must first come to have and attribute propositional attitudes, they must learn how propositional attitudes - e.g., beliefs, desires, and emotions - combine to form reasons for action, and through exposure to folk psychological narratives they learn the norms of acting for reasons. Only after we master natural language and become proficient in understanding and providing folk psychological narratives can we learn what is now commonly referred to as mindreading, i.e. explaining and predicting behavior on the basis of attributed mental states. But even then, mindreading is a rarely used, specialized skill. We mindread only when our primary modes of social cognition break down, i.e., only when embodied practices are ambiguous and we cannot understand an agent's behavior in terms of familiar social norms and behavioral scripts.

Because NPH holds that we develop our sophisticated social cognition skills by comprehending folk psychological narratives, it implies that children could not even be candidate mindreaders until after they master natural language. Thus, there is a chasm between the preverbal social cognition of infants and that of older children and adults. On this view, only those who have mastered language and encountered folk psychological narratives are capable of mindreading. Non-linguistic and pre-linguistic beings' understanding of others is limited to non-propositional, non-representational, and nonmental understanding (Hutto, 2008, p. Ch. 3). Although NPH is not the only option for embodied cognition, many embodied cognition theorists accept the idea that children are not capable of mindreading until after they master natural language (Gallagher \& Zahavi, 2008; Ratcliffe, 2007).

This idea allegedly is bolstered by results in developmental psychology. For much of the last 30 years, the standard developmental picture of theory of mind has been that at around 4 years of age, children undergo a fundamental shift in their theory of mind abilities. As Heinz Wimmer and Josef Perner's experiments first revealed, and other experiments have since replicated, before the age of 4 children cannot pass standard falsebelief tasks (Gopnik \& Astington, 1988; Wimmer \& Perner, 1983). In one task commonly referred to as the Sally-Anne task, children listen to a story as it is enacted with dolls named Sally and Anne. In the scene, Sally hides a toy in one place and then she leaves the scene. Anne moves the toy from the original hiding place to a new hiding place. When children 
younger than 4 years old are asked where Sally will look for the toy, they answer incorrectly. They say she will look in the new hiding place. Children 4-years and older, however, typically answer correctly. They say Sally will look in the original place and give appropriate explanations for why she will look there. This evidence has been taken to show that there is a significant developmental shift in theory of mind abilities at around 4 years of age. At age 4 children shift from lacking proficiency with the concept of belief to being able to appropriately apply the concept in a range of situations. That is, at age 4 children master the belief concept. Given that the concept of belief plays an important role in understanding others' mental states, the standard false-belief task has been taken to be the measuring stick of theory of mind abilities.

Embodied cognition theorists do not regard the standard false-belief task as evidence that theory of mind is developmentally fundamental or our primary mode of social cognition. They argue that the experimental setup is not ecologically valid; it does not test our social cognition in ordinary interactions. Nevertheless, they do regard it as evidence for their developmental timeline because it explicitly requires the subject to attribute propositional attitudes to explain and predict the target's behavior. Embodied cognition theorists argue that children must first master language and become proficient in understanding and providing folk psychological narratives. Only after these developments can children develop the ability to explain and predict behavior on the basis of propositional attitude ascriptions. Thus, despite the mistaken assumptions about the importance of the cognitive skills tested in the standard false-belief task, the fact that children first pass this task around the same age that they master the forms and norms folk psychological narratives is evidence for the developmental timeline suggested by embodied cognition.

Embodied cognition theorists take the results of the standard false-belief task as evidence for their claim that children are not even capable of mindreading until fairly late in development, which suggests that theory of mind is not developmentally fundamental. What is developmentally fundamental is embodied intersubjectivity. Embodied intersubjectivity, along with knowledge of social norms and behavioral scripts, make up our ordinary social cognitive interactions even as adults.

\section{Challenges for Embodied Cognition}

In this section, I identify three challenges for embodied accounts of social cognition. ${ }^{2}$ The first problem is that they embodied cognition rely on outdated empirical data. Second, they leave an un-bridged gap between the kind of social cognitive capacities of preverbal children and the social cognitive capacities of older children and adults. Third, embodied theories of social cognition focus exclusively on "online" cognitive processes, thereby neglecting the legitimate and important role of "offline" cognitive processes. None of these challenges is insurmountable, but I do take them to be serious shortcomings that embodied cognition must address.

\subsection{Outdated Developmental Timeline}

${ }^{2}$ See Spaulding (2012) for a guide to discussions about embodied social cognition. 
As I pointed out above, embodied social cognition acquires evidence for its developmental timeline from the results of the standard false-belief task. However, the standard falsebelief task is no longer regarded as a good test for theory of mind abilities. Paul Bloom and Tim German persuasively argue that passing the standard false-belief task is neither necessary nor sufficient for theory of mind. The standard false-belief task tests for a variety of general cognitive skills that are not specifically theory of mind skills, and explicit reasoning about false beliefs is not necessary for theory of mind (Bloom \& German, 2000).

Kristine Onishi and Renée Baillargeon (2005) object to the standard false-belief tasks, arguing that these tasks are computationally and linguistically too taxing for children younger than 4 years old. The standard false-belief task requires children to remember the details of the story, who saw what and when, to interpret adults' questions, and give appropriate responses to these questions. Many of these task demands are unrelated to theory of mind per se. Rather, the demands of the standard false-belief task reveal performance of executive functions, e.g., memory and response inhibition. In lieu of the standard measuring stick, Onishi and Baillargeon opt for a simplified non-linguistic falsebelief task to measure theory of mind abilities of younger children.

In their novel non-linguistic false-belief task, 15-month-old infants watch an actor put a toy watermelon slice in one of two adjacent boxes, a green box or yellow box. Next, the toy is moved. In half the trials the toy is moved halfway to the other box and then back to the original box, and in the other half of the trials the toy is moved to the other box. For both of these conditions the actor either does or does not see the movement of the toy. (In one variation she looks through an opening in the tops of the boxes, and in another variation she does not.) Using the violation-of-expectation method, Onishi and Baillargeon found that 15-month-old infants looked longer in two cases: first, when the actor does not see that the toy's location has changed, but searches in the correct box anyway, and second, when the actor does see the toy being relocated but the actor reaches in the incorrect box.

Onishi and Baillargeon interpret these results as showing that the 15-month-old infants expect the actor to search for the toy on the basis of her belief about the toy's location. When the actor does not search for the toy on the basis of her belief, the infants' expectations are violated and they thus looked longer at those events. Onishi and Baillargeon take this to be good evidence for the conclusion that 15-month-old infants already have mindreading abilities and that the ability to mindread, in at least a rudimentary form, is innate.

Onishi and Baillargeon were the first to use non-linguistic methods for testing falsebelief understanding. Since the publication of their article, numerous studies employing a variety of non-verbal testing methods - anticipatory looking, violation of expectation, and active helping - have found evidence that preverbal infants are sensitive to others' intentions, perceptions, perspectives regarding objects, intentions in pretend scenarios, etc. (Gergely, Bekkering, \& Kirly, 2002; Luo \& Baillargeon, 2007; Onishi, Baillargeon, \& Leslie, 2007; Song \& Baillargeon, 2007). For example, Baillargeon and colleagues found that 5month-old infants expect agents to act according to their preferences, and they looked longer when agents act contrary to their exhibited preferences.

"[A]fter watching familiarization events in which an agent repeatedly grasps object$\mathrm{A}$, infants look longer at test events if the agent now grasps object-B, but only if object- $\mathrm{B}$ is both present and visible to the agent during the familiarization events, so 
that infants have evidence that the agent prefers object-A over object-B. These different looking-patterns indicate that infants do not merely form associations but consider (at the very least) the motivational and reality-incongruent informational states that underlie agents' actions" (Baillargeon, Scott, \& He, 2010, p. 115).

This study, and dozens others like it, purport to show that children are sensitive to others' mental states long before they can pass the standard false-belief task. The standard falsebelief task, it is argued, tests for children's ability to express linguistically what they are capable of understanding long before age 4 .

Given that embodied cognition theorists are committed to the idea that children are not capable of mindreading until after they master natural language and become proficient with folk psychological narratives, they must reject these empirical findings. They must argue that we can explain the infants' behaviors without attributing to them precocious mindreading abilities. Their task is to reinterpret the results in terms of non-mentalistic behavior-based explanations, e.g., infants learn the behavioral rule that "agents look for an object where they last saw it."

Currently, there is a vigorous debate about how to understand the results of these non-linguistic mindreading tasks. Some argue that these studies show that infants understand false beliefs and perhaps already possess the belief concept. According to this interpretation, the reason children fail the standard false-belief task until age 4 is that up until age 4 the task is too demanding on younger children's executive system, e.g., shortterm memory and response-inhibition (Baillargeon et al., 2010; Leslie, Friedman, \& German, 2004; Onishi \& Baillargeon, 2005). Others argue that these studies show that infants must be very clever behavior readers because it is not possible for infants to understand false beliefs or possess the belief concept. On this interpretation, children 4years and younger fail the standard false- because up until age 4 they do not fully grasp the belief concept (Perner \& Ruffman, 2005).

Embodied cognition is committed to the latter interpretation of these studies. Thus, they must argue for a reinterpretation of the results of the studies in terms of behavioral rules and associations. Unfortunately, however, embodied cognition theorists do not provide such a reinterpretation. They mostly ignore these findings because they regard them as tainted by the presupposition that mindreading is a fundamental part of our ordinary social cognitive interactions. This is unfortunate for two reasons.

First, there is a growing body of evidence suggesting that infants really are capable of more than just non-mentalistic behavior reading. This body of evidence is growing in terms of the diversity of experimental paradigms employed and the number of studies finding that infants are sensitive to others' mental states. As these findings become more robust, explaining away these results in terms of ad hoc behavioral associations and rules becomes less appealing. These experimental results make the developmental timeline of the standard false-belief task look much less plausible, which is bad news for embodied cognition's account of social cognition.

Second, although embodied cognition theorists may be right that these studies presuppose that mindreading is a fundamental part of our ordinary social interactions, this does not mean they should simply ignore these findings. Embodied cognition theorists ought to explain what they think really is happening in these studies and how we should understand these results. Otherwise, embodied cognition is open to the obvious objection 
that it is in conflict with much of the contemporary data from developmental psychology. Thus, the first challenge that embodied cognition faces is to justify its reliance on a developmental timeline that appears to have been empirically falsified.

\subsection{Cognitive Gap}

Suppose that embodied cognition's developmental timeline is correct, and we can explain adequately all of the results of the new wave, non-linguistic mindreading tests in terms of non-mentalistic embodied cues and behavioral rules and associations. If this is the case, then infants and young children do not, indeed cannot, explain and predict behavior on the basis of mental state attributions. In other words, preverbal children cannot mindread. Older children and adults, however, can mindread. Thus, there is a cognitive gap between the social cognitive capacities of preverbal children and the social cognitive capacities of older children and adults. Even if mindreading is rare for older children and adults, embodied cognition needs an account of how we develop the capacity for mindreading from purely non-mentalistic embodied cues (Wilby, 2012).

A comprehensive account of social cognition must describe our social cognitive capacities as infants and young children, our social cognitive capacities as older children and adults, and how we develop the latter from the former. Embodied cognition theorists focus on denying that infants and young children can mindread and denying that mindreading is prevalent even in adults, the result of which is that they neglect to explain how we develop this capacity for mindreading. This gap is problematic because it is not obvious how a capacity for mindreading could develop from purely non-mentalistic embodied practices. Embodied cognition theorists often cite the mastery of natural language as the basis for developing the capacity for mindreading. But this is not an explanation; it is a placeholder for an explanation. To have a comprehensive account, embodied cognition must explain how the mastery of natural language enables children to develop the capacity for mindreading. ${ }^{3}$

NPH may offer the most promising account of how children develop the capacity for mindreading. Once children master natural language and become proficient with the forms and norms of folk psychological narratives, they can begin to understand how beliefs and desires interact to form reasons for actions. Once they understand reasons for actions, they learn to explain and predict behavior in terms of these reasons for actions. The problem with this account is that it seems to have the story backwards (Spaulding, 2011; Thompson, 2012). On the face of it, one could not understand folk psychological narratives without already understanding how mental states cause behavior. Understanding the folk psychological aspect of folk psychological narratives seems to presuppose a capacity for mindreading. To face this challenge, embodied cognition proponents must show that mastering folk psychological narratives does not presuppose a capacity for mindreading.

\subsection{Neglect of Offline Processes}

The final challenge for embodied cognition concerns the comprehensiveness of the account. Embodied cognition focuses exclusively on the role of online processes in social cognition

${ }^{3}$ See Wilby (2012) for an evaluation of various attempts to close this cognitive gap. 
(de Bruin \& Kästner, 2012). Online processes are cognitive processes that involve responding directly to rather than representing features of the environment. Embodied cognition's notion of embodied intersubjectivity illustrates this well. Embodied intersubjectivity consists in primary and secondary intersubjectivity: facial imitation and proprioceptive sense of one's body, the capacity to detect and track eye movement, to detect intentional behavior, and to read emotions from actions and expressive movements of others, and joint attention. Embodied cognition regards these as online, nonrepresentational cognitive processes aims to explain our social interactions in terms of these online processes.

Online processes are an important element of social cognition, of course. Embodied cognition is surely right that these online processes are developmentally fundamental. An account of social cognition would be inadequate if it failed to include a role for online processes such as facial imitation, tracking eye movement, and detecting intentional behavior. Embodied cognition theorists allege that theory of mind accounts are guilty of neglecting the role of online processes and focusing exclusively on offline processes.

In contrast to online processes, offline processes involve internal representations, which are not bound to the current features of the agent's body or her environment, e.g., propositional attitude ascriptions. Theory of mind accounts assume that the mind is an intracranial information-processing system. On this view, cognition is a computational process of manipulating symbolic representations. Theory of mind accounts focus on our ability to explain and predict others' behaviors in terms of mental state attributions. They explain these social cognitive capacities by adverting to computational processes, modular mechanisms, internal representations, and so on. Given the emphasis on these offline computational processes, these accounts tend to neglect the role of online processes in social cognition. Embodied cognition rejects the idea assumed in the philosophical and empirical literature on theory of mind that social cognition primarily involves offline processes. Embodied cognition theorists regard this assumption as false and aim to show that social cognition is best explicable in terms of online processes.

Of course, we employ both online and offline cognitive processes in our social interactions. Social cognition requires both responding directly to features of the environment and manipulating information that is absent from the environment and so has to be internally represented. Embodied cognition theorists do not deny this. They do not regard offline cognitive processes as impossible. They simply regard them as relatively unimportant. And this is a mistake. In many cases, it is beneficial to withdraw from the immediate surroundings so as not to automatically act upon particular affordances. Think of the enormous cognitive benefit the capacity to engage in counterfactual reasoning affords. Instead of simply engaging directly with the environment, the agent may consider other ways to respond to the environment, consider hypothetical action plans, evaluate various means of achieving one's goals, etc.

Moreover, the separation of online and offline processes is artificial. An agent's online and offline processes interact to yield cognitive flexibility and autonomy from environmental stimulation such that the agent becomes less dependent upon, and gains new ways of relating to, her environment and other agents in her environment. For example, when we perceive someone blushing, we may take into consideration what immediately happened that could have caused the blush, this person's recent history and personality, and social knowledge about what causes people to blush. Inferring that the 
person is blushing because she is angry gives rise to different interaction than inferring that she is embarrassed. And inferring that she is embarrassed about something she said yields a different sort of online interaction than inferring that she is embarrassed by something you said. When we perceive the blush, we automatically take into consideration background information, and the background information influences how we perceive the situation. In order to be a comprehensive account of social cognition, embodied cognition must recognize the legitimate and important role such offline processes play in social cognition. ${ }^{4}$

\section{Conclusion}

Embodied cognition's deflationary account is a welcome challenge to the theory of mind orthodoxy. It prompts traditional theory of mind accounts to justify the assumption that we must and often do attribute propositional attitudes to others in order to understand and interact with them. It spurs theory of mind advocates to consider the neglected role of embodied practices and online processes. Consideration of this deflationary account of social cognition brings to light assumptions of the theory of mind literature that need reconsideration. Embodied cognition encourages us to scrutinize the presuppositions and conclusions of the empirical literature on social cognition.

Embodied cognition provides a different paradigm for studying social cognition. To provide a comprehensive, adequate account of social cognition, embodied cognition needs to answer the challenges described above. This requires considering the new wave falsebelief tasks and other non-linguistic theory of mind tasks. Embodied cognition proponents need to respond to the objection that their account relies on an empirically disconfirmed developmental timeline and provide an alternative explanation of these empirical results. They must explain how exactly sophisticated social cognition develops from nonmentalistic embodied practices. And they must make room in their account for the legitimate and important role of offline processes in social cognition.

It is an open question whether embodied cognition or theory of mind offers a more compelling account of social cognition. My own view is that embodied cognition has some distance to go before we can declare it the superior account. It is clear, however, that the debate about social cognition has benefited greatly from this clash of paradigms. Embodied cognition highlights genuine shortcomings and questionable assumptions of theory of mind and provides a radically different alternative account of social cognition. The introduction of this radically different account has revived stagnant debates and spurred reevaluation of old theory of mind dogmas. This clashing of paradigms is the best way forward for the debate about social cognition.

\section{References:}

Baillargeon, R., Scott, R., \& He, Z. (2010). False-belief understanding in infants. Trends in Cognitive Sciences, 14(3), 110-118.

\footnotetext{
${ }^{4}$ See de Bruin and Kästner (2012) for an extended defense of this argument and a positive account of how online and offline social cognition processes dynamically interact.
} 
Bloom, P., \& German, T. P. (2000). Two reasons to abandon the false belief task as a test of theory of mind. Cognition, 77(1), 25-31.

de Bruin, L. C., \& Kästner, L. (2012). Dynamic embodied cognition. Phenomenology and the Cognitive Sciences, 11(4), 541-563.

Gallagher, S. (2005). How the Body Shapes the Mind: Oxford University Press, USA.

Gallagher, S., \& Hutto, D. D. (2008). Understanding others through primary interaction and narrative practice. In Sinha, Itkonen, Zlatev \& Racine (Eds.), The Shared Mind: Perspectives on Intersubjectivity. Amsterdam: John Benjamins.

Gallagher, S., \& Zahavi, D. (2008). The Phenomenological Mind: An Introduction To Philosophy of Mind and Cognitive Science. New York: Routledge.

Gergely, G., Bekkering, H., \& Kirly, I. (2002). Developmental psychology: Rational imitation in preverbal infants. Nature, 415(6873), 755-756.

Gopnik, A., \& Astington, J. W. (1988). Children's understanding of representational change and its relation to the understanding of false belief and the appearance-reality distinction. Child Development, 59(1), 26-37.

Hutto, D. D. (2008). Folk Psychological Narratives: The Sociocultural Basis of Understanding Reasons. Cambridge, MA: MIT Press.

Leslie, A. M., Friedman, O., \& German, T. P. (2004). Core mechanisms in 'theory of mind'. Trends in Cognitive Sciences, 8(12), 528-533.

Luo, Y., \& Baillargeon, R. (2007). Do 12.5-month-old infants consider what objects others can see when interpreting their actions? Cognition, 105(3), 489-512.

Onishi, K. H., \& Baillargeon, R. (2005). Do 15-month-old infants understand false beliefs? Science, 308(5719), 255-258.

Onishi, K. H., Baillargeon, R., \& Leslie, A. M. (2007). 15-month-old infants detect violations in pretend scenarios. Acta Psychologica, 124(1), 106-128.

Perner, J., \& Ruffman, T. (2005). Infants' insight into the mind: How deep? Science, 308(5719), 214.

Ratcliffe, M. (2007). Rethinking Comonsense Psychology. New York: Palgrave Macmillan.

Song, H., \& Baillargeon, R. (2007). Can 9.5-month-old infants attribute to an agent a disposition to perform a particular action on objects? Acta Psychologica, 124(1), 79105.

Spaulding, S. (2010). Embodied cognition and mindreading. Mind \& Language, 25(1), 119140.

Spaulding, S. (2011). A critique of embodied simulation. Review of Philosophy and Psychology, 2(3), 579-599.

Spaulding, S. (2012). Introduction to debates on embodied social cognition. Phenomenology and the Cognitive Sciences, 11(4), 431-448.

Thompson, J. (2012). Implicit mindreading and embodied cognition. Phenomenology and the Cognitive Sciences, 11(4), 449-466.

Wilby, M. (2012). Embodying the false-belief tasks. Phenomenology and the Cognitive Sciences, 11(4), 519-540. 
Wimmer, H., \& Perner, J. (1983). Beliefs about beliefs: Representation and constraining function of wrong beliefs in young children's understanding of deception. Cognition, 13(1), 103-128. 